\title{
Water-Stable Cathode for High Rate Na-Ion
}

\section{Batteries}

Yi Zhang $f^{1}$, Miaomiao Wu $\neq^{1}$, Wei Teng ${ }^{2}$, Jiwei Ma ${ }^{1}$, Renyuan Zhang ${ }^{1 *}$ and Yunhui Huang ${ }^{1 *}$

1. Institute of New Energy for Vehicles, Shanghai Key Laboratory for R\&D and Application of Metallic Functional Materials, School of Materials Science and Engineering, Tongji University, Shanghai, 201804, P. R. China

2. State Key Laboratory for Pollution Control, School of Environmental Science and Engineering, Tongji University, Shanghai 200092, P. R. China.

\section{E-mail: *ryzhang@tongji.edu.cn; *huangyh@hust.edu.cn}

Characterizations: ${ }^{7} \mathrm{Li}$ NMR spectra was acquired on a Bruker Advance $400 \mathrm{MHz}$ spectrometer by using a $3.2 \mathrm{~mm}$ MAS probe with a magic-angle spinning frequency of $20 \mathrm{kHz}$. SEM images and EDS mapping were obtained on JEOL JSM-6390 microscope (Japan). TEM images were acquired by a Hitachi HT-7700 transmission electron microscope (Japan) operating at $100 \mathrm{kV}$. High-resolution TEM (HRTEM) micrographs were obtained with a Philips Tecnai F20 FEG-TEM (The USA) operated at $200 \mathrm{kV}$. Samples for TEM analysis were prepared by drying a drop of cyclohexane solution containing the materials on the surface of a carbon-coated 
copper grid. The X-ray diffraction XRD patterns were obtained using a Rigaku D/MAX-RB (Japan) with monochromatized $\mathrm{Cu} \mathrm{K} \alpha$ radiation $\left(\lambda=1.5418 \AA\right.$ ) in the $2 \theta$ ranging from $10^{\circ}$ to $70^{\circ}$. For ex-situ XRD measurements, coin cells were prepared and charged/discharged to the target potential, then these cells were disassembled in argon-filled glovebox. All electrodes were taken out from coin cells and washed with dimethyl carbonate (DMC) for three times. After standing for $12 \mathrm{~h}$, all electrodes were sealed by Kapton tape for ex-situ XRD test. All powder patterns were refined using the Rietveld method as implemented in the GSAS program. 1 The crystal structure were visualized by VESTA 2 and $3 \mathrm{ds}$ Max software. In this work, we utilized Vienna Ab initio Simulation Package (VASP) ${ }^{3-6}$ to perfrom DFT within periodic models. Generalized gradient approximation $(\mathrm{GGA})^{7}$ with projector augmented wave $(\mathrm{PAW})^{8}, \underline{9}$ method was applied in calculations to better simulate the electronic structures and the core-electron interaction. The cutoff energy is set to be $500 \mathrm{eV}$, and the force threshold of optimization convergence is $0.05 \mathrm{eV}$ $\AA^{-1}$. Here we employed Hubbard $U$ potential to describe the Coulomb electron interaction precisely. We followed $\mathrm{U}$ and $\mathrm{J}$ values from previous literatures, $\underline{10}$ and $\mathrm{U}$ values of $\mathrm{Mn}, \mathrm{Ni}, \mathrm{Fe}$ and $\mathrm{Co}$ were $5 \mathrm{eV}, 6 \mathrm{eV}, 6 \mathrm{eV}$ and $2.9 \mathrm{eV}$, respectively. $\mathrm{J}$ values of $\mathrm{Mn}, \mathrm{Ni}, \mathrm{Fe}$ were set to be 1 $\mathrm{eV}$; J value of Co was $0 \mathrm{eV}$. Spin polarization effect was considered in this work, and Van-derWaals interaction along the Z-axis was involved in calculation. Structural optimizations were performed until the residual forces on each ion converged to smaller than $0.05 \mathrm{eV} \AA^{-1}$. According to the experimental lattice of $\mathrm{Na}_{0.66} \mathrm{Ni}_{0.33} \mathrm{Mn}_{0.66} \mathrm{O}_{2}$, we built a (001) surface model with $2 \times 2$ supercell, and the vacuum-layer length was more than $12 \AA$ to avoid interaction of mirror slabs. A surface $\mathrm{Ni}$ atom was substituted to be $\mathrm{Fe}, \mathrm{Co}$ and $\mathrm{Ni}$ atom to simulate the corresponding hetero-atom doping conditions. The Brillouin zone were meshed using $4 \times 4 \times 1$ Monkhorst-Pack 
grid 11 for all of the structural relaxations. The adsorption energies of $\mathrm{H}_{2} \mathrm{O}\left(\Delta E_{\mathrm{ads}}\right)$ on the considered slabs are defined as:

$$
\Delta E_{\mathrm{ads}}=E_{\mathrm{slab}}+\mathrm{H} 2 \mathrm{O}-E_{\mathrm{slab}}-E_{\mathrm{H} 20}
$$

where $\Delta E_{\text {slab }}$ means the total energy of slab and $\mathrm{H}_{2} \mathrm{O}, E_{\text {slab }}$ denotes the slab energy and $E_{\mathrm{H} 2 \mathrm{O}}$ represents the energy of $\mathrm{H}_{2} \mathrm{O}$.

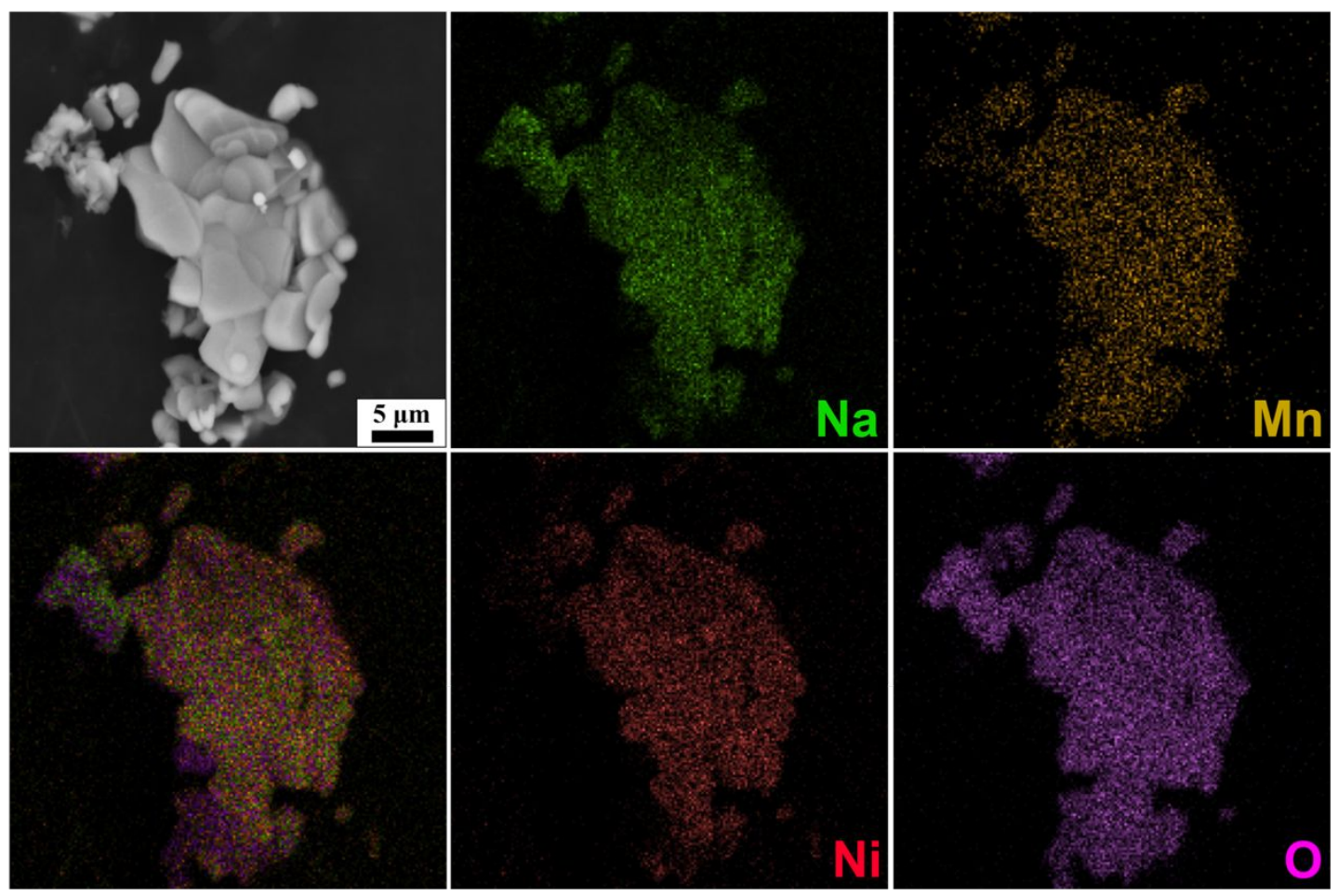

Figure S1. EDX elemental mappings of NNM sample. 


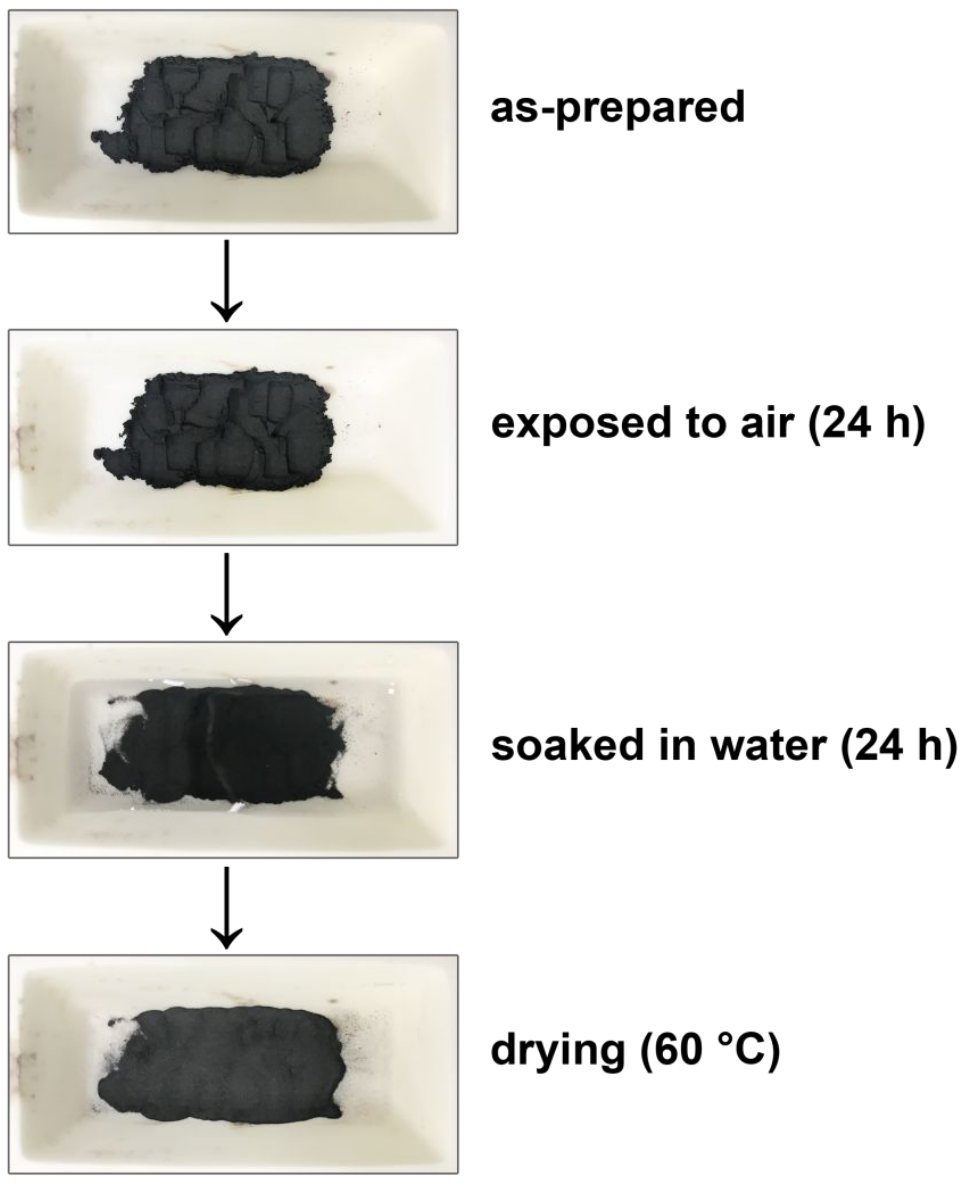

Figure S2. Schematic of the water soaking treatment for NNM, NCNM, NFNM and NLNM samples. 

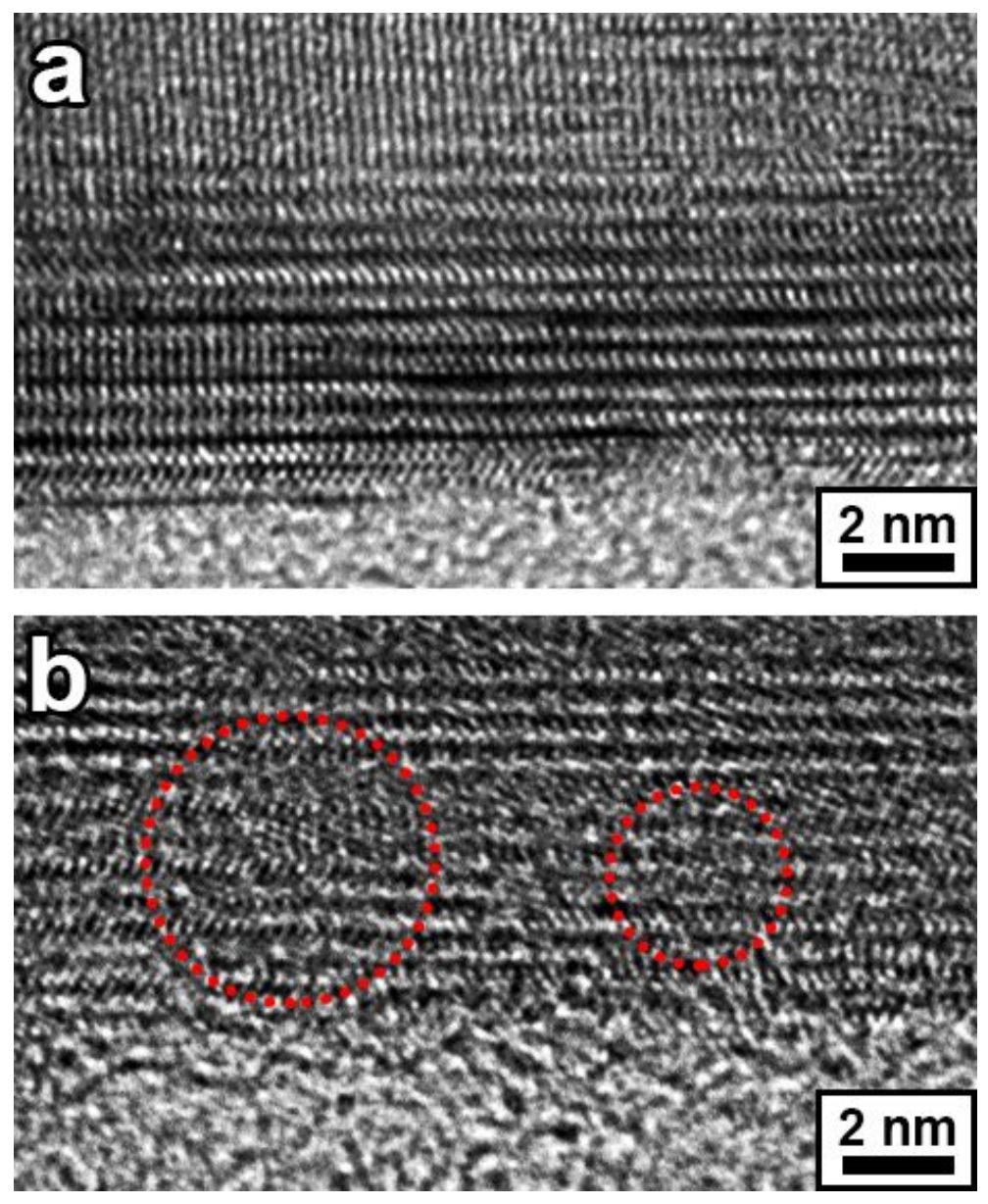

Figure S3. (a) HRTEM image of NFNM sample before water soaking. (b) HRTEM image of NFNM sample after water soaking. The red zone represent the dameged area, which is destroyed by water insertion. 

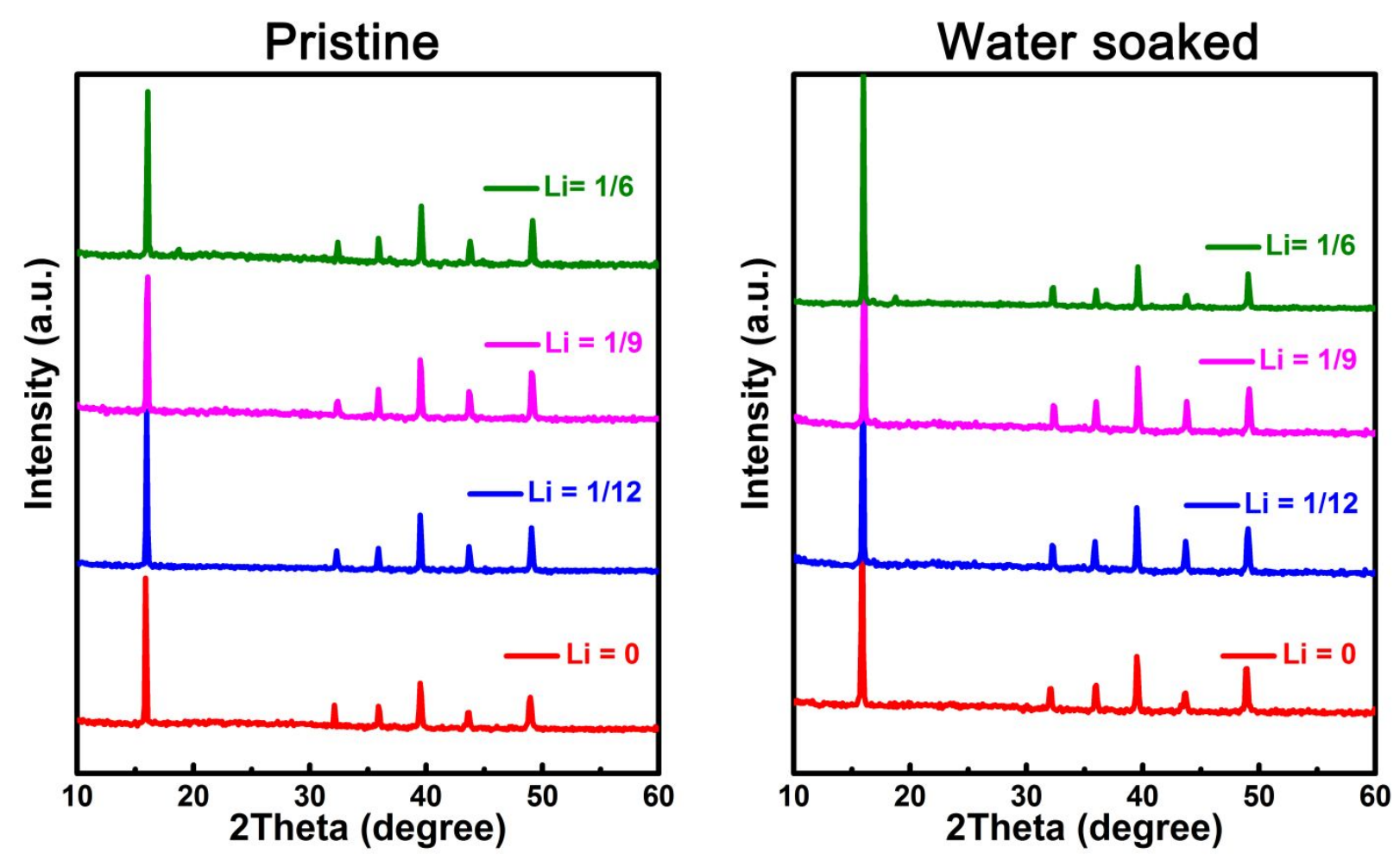

Figure S4. (a) XRD patterns with different doping amount of $\mathrm{Li}^{+}$at pristine state. (b) XRD patterns with different doping amount of $\mathrm{Li}^{+}$after water-soaking treatment. 
(a)

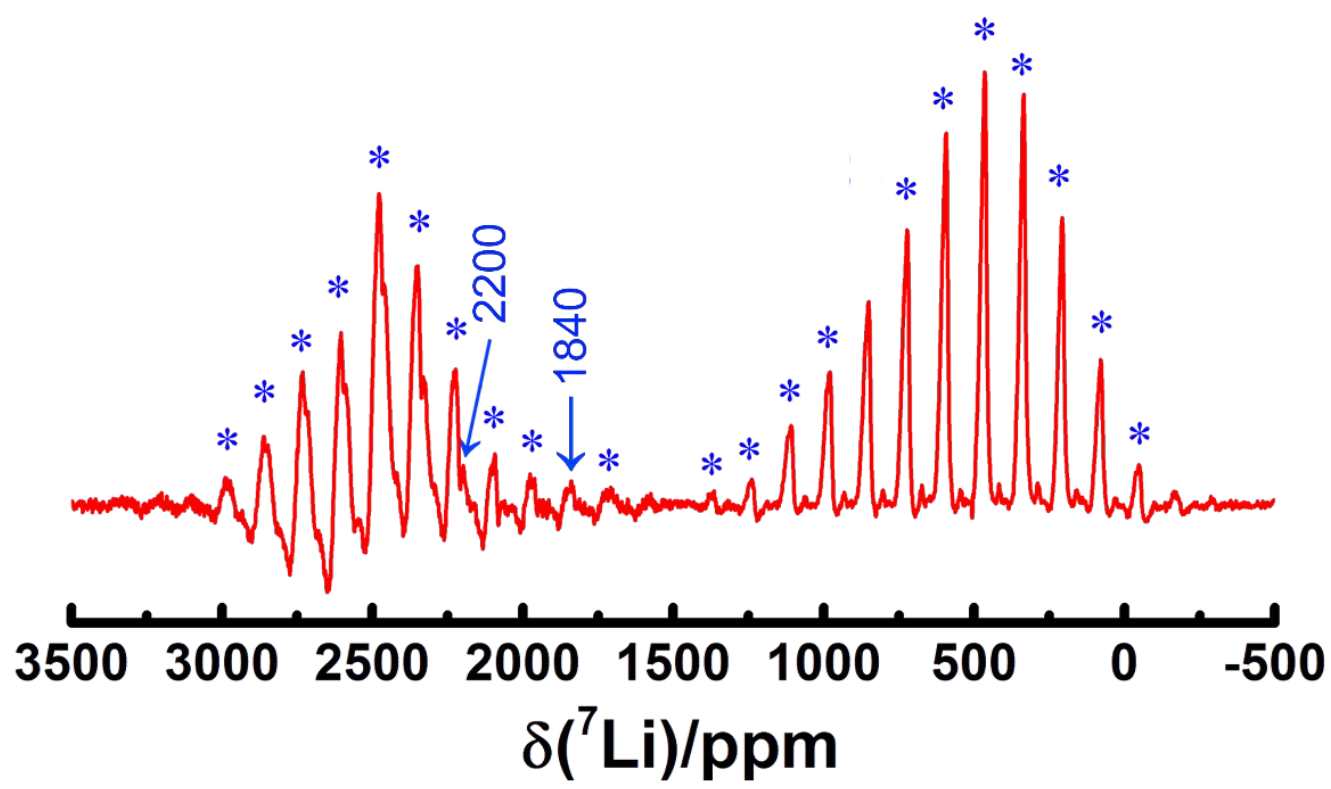

(b)

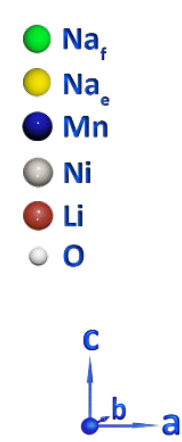

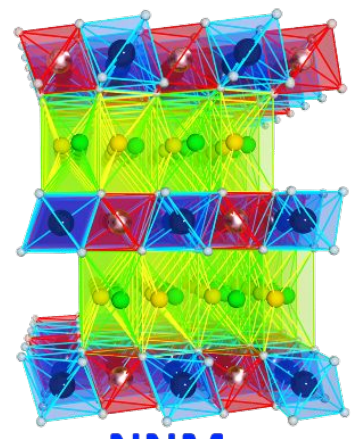

NNM

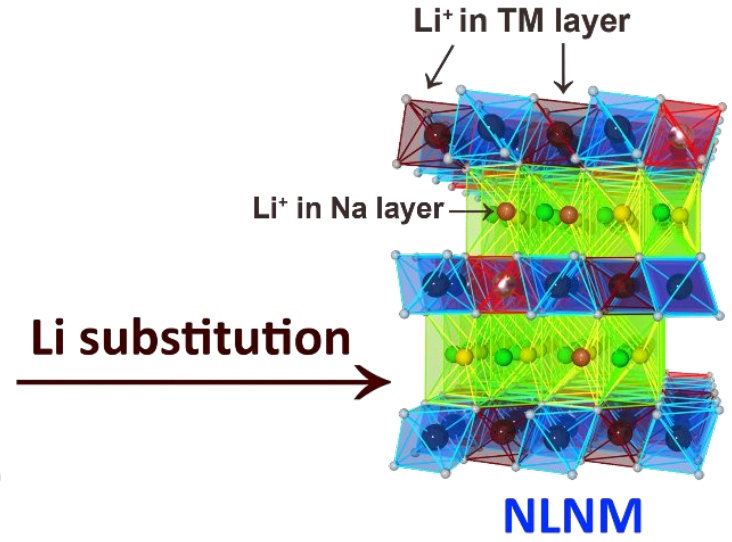

Figure S5. (a) ${ }^{7} \mathrm{Li}$ solid-state NMR spectrum of NLNM sample recorded at $400 \mathrm{MHz}$, the * denotes spinning sidebands, the peaks at 1840 and 2200 can be assigned to $\mathrm{Li}^{+}$in the octahedral site within the TM layer. (b) Schematic of $\mathrm{Li}^{+}$location in NLNM sample. 

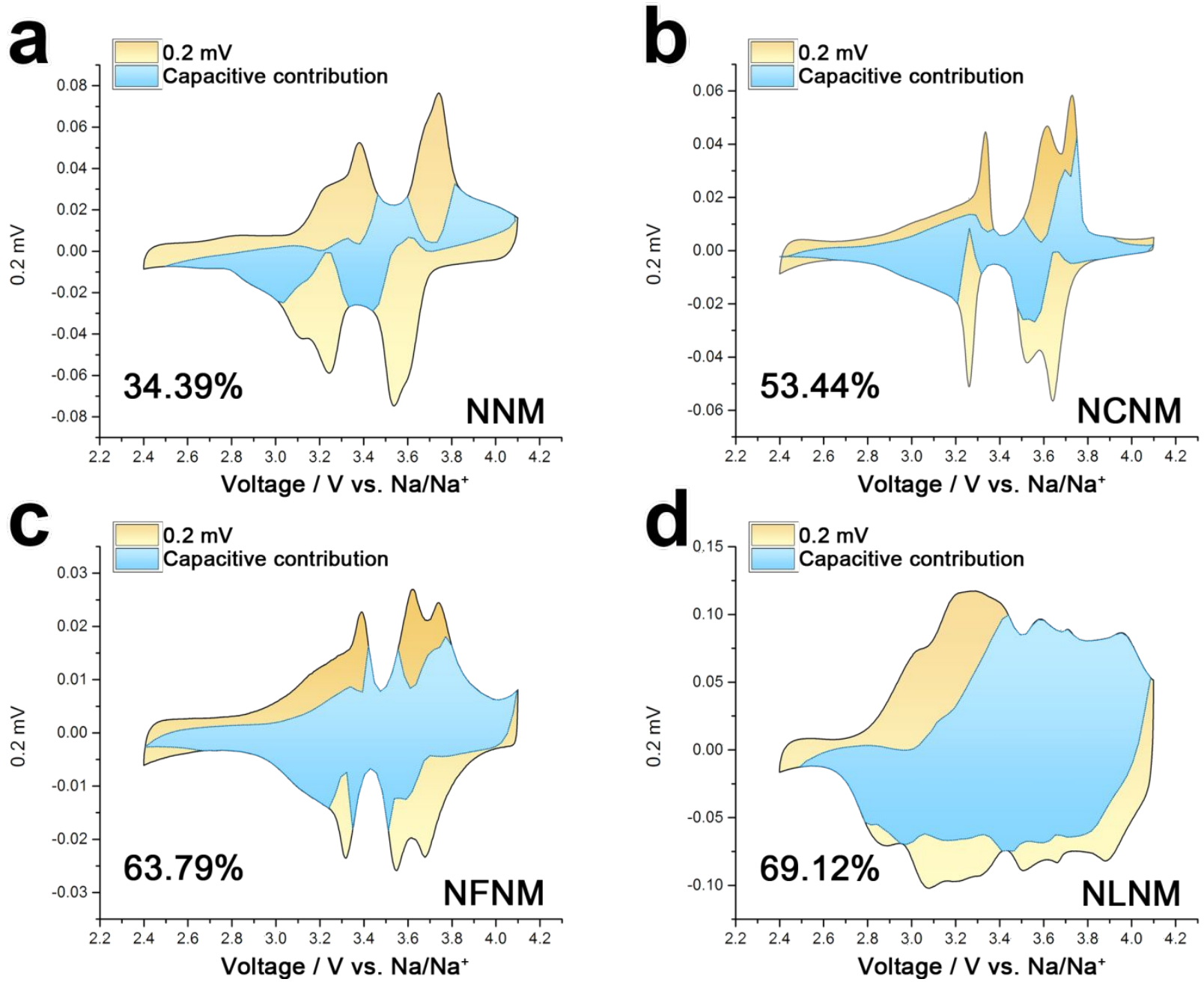

Figure S6. The capacitive contribution of a). NNM; b). NCNM; c). NFNM and d). NLNM electrodes. 

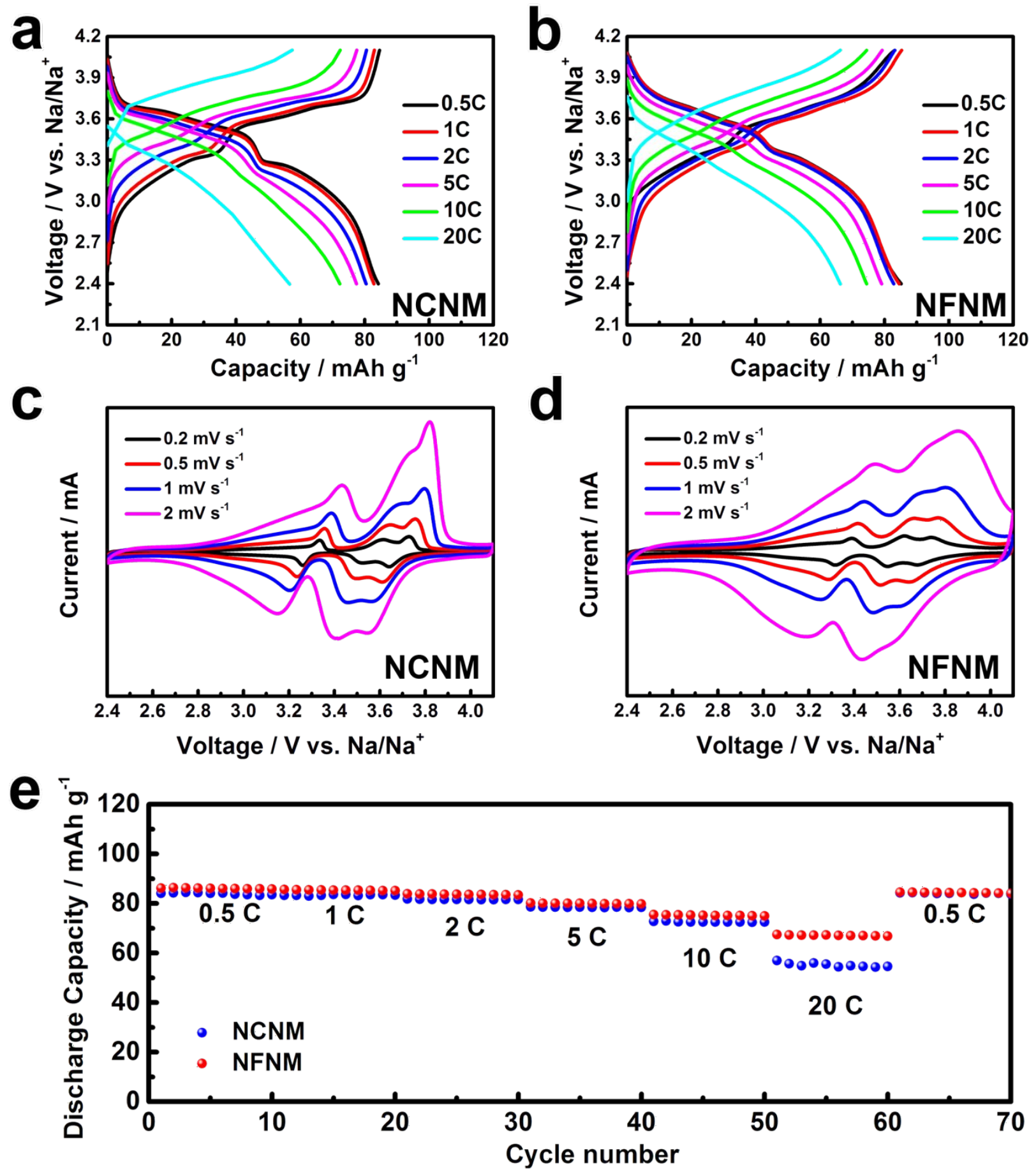

Figure S7. (a, b) charge/discharge profiles, (c, d) CV curves and (e) rate performance of NCNM and NFNM samples. 
Table S1. Crystallographic parameters of NNM, NCNM, NFNM and NLNM samples refined by the Rietveld methode.

\begin{tabular}{ccccccc}
\hline Sample & $\mathrm{a}(\AA)$ & $\mathrm{b}(\AA)$ & $\mathrm{c}(\AA)$ & $\mathrm{V}\left(\AA^{3}\right)$ & $\mathbf{R}_{\mathrm{p}}$ & $\mathbf{R}_{\mathrm{wp}}$ \\
\hline NNM & 2.886953 & 2.886953 & 11.154008 & 80.508 & $3.24 \%$ & $5.46 \%$ \\
NCNM & 2.894620 & 2.894620 & 11.169846 & 81.051 & $2.56 \%$ & $4.26 \%$ \\
NFNM & 2.905048 & 2.905048 & 11.182226 & 81.727 & $2.7 \%$ & $5.08 \%$ \\
NLNM & 2.889076 & 2.889076 & 11.121620 & 80.393 & $4.52 \%$ & $6.25 \%$ \\
\hline
\end{tabular}

Table S2. Slab thickness, d-spacing of Na layered interslab distance by the Rietveld methode.

\begin{tabular}{ccccc}
\hline Samples & NNM & NCNM & NFNM & NLNM \\
\hline TMO $_{2}(\AA)$ & 2.097 & 2.112 & 2.121 & 2.103 \\
d-spacing $(\AA)$ & 3.48 & 3.473 & 3.47 & 3.457 \\
\hline
\end{tabular}




\section{References}

1. B. H. Toby, J. Appl. Crystallogr., 2001, 34, 210-213.

2. K. Momma and F. Izumi, J. Appl. Crystallogr., 2008, 41, 653-658.

3. G. Kresse and J. Hafner, Phys. Rev. B, 1994, 49, 14251-14269.

4. G. Kresse and J. Hafner, Phys. Rev. B, 1993, 47, 558-561.

5. G. Kresse and J. Furthmüller, Comp. Mater. Sci., 1996, 6, 15-50.

6. G. Kresse and J. Furthmüller, Phys. Rev. B, 1996, 54, 11169-11186.

7. J. P. Perdew, K. Burke and M. Ernzerhof, Phys. Rev. Lett., 1996, 77, 3865-3868.

8. G. Kresse and D. Joubert, Phys. Rev. B, 1999, 59, 1758-1775.

9. P. E. Blöchl, Phys. Rev. B, 1994, 50, 17953-17979.

10. L. Li, L. Zhu, L.-H. Xu, T.-M. Cheng, W. Wang, X. Li and Q.-T. Sui, J. Mater. Chem. A, 2014, 2, 4251-4255.

11. H. J. Monkhorst and J. D. Pack, Phys. Rev. B, 1976, 13, 5188-5192. 